\title{
Rates and Risk-Factors of Post Implantation Infection in Cardiac Implantable Electronic Devices
}

\author{
David Karnani ${ }^{1}$, T. Eric White ${ }^{2}$ \\ ${ }^{1}$ Indiana University School of Medicine, ${ }^{2}$ Parkview Health, Department of Cardiology
}

\section{Background/Hypothesis:}

Cardiac implantable electronic devices (CIED) include permanent pacemakers (PPM), implantable cardioverter defibrillators (ICD), and cardiac resynchronization therapy (CRT). Both ICDs and dual chamber PPMs are two-lead systems; whereas, CRT is a three-lead system. Indications for CIEDs include arrythmia, risk of sudden cardiac death and AV block. From 1993 to 2003, over 4 million CIEDs were implanted. With the upward trend in the rate of CIED implantations, the post-operative infection burden has increased concurrently. Device related infections are a serious complication of CIED implants. They are associated with a significant increase in the rate of mortality as well as a significant increase in financial burden. We hypothesize two-lead systems will have significantly lower rate of infection than three-lead devices

\section{Methods:}

This study will be a retrospective review of patient charts who are hospitalized in the Parkview Health medical system in Fort Wayne, Indiana over a three-year period. All patients who have received a CIED during that time frame will be included in the study. Patients will be categorized by specific implant type and recorded as developing post-operation infection or not.

Demographic and health status data will also be collected and analyzed for correlation with the development of device related infection.

\section{Results:}

Our results will compare the device related infection rate for two-lead versus three lead systems, as well as the infection rate for each type of CIED. We also expect to find results which support a correlation between specific patient demographics and patient health factors.

\section{Conclusion and Impact:}

The results of this study will serve as a guide for identifying high risk procedures and patients. The objective is to provide providers with guidelines for the use of post-operative prophylaxis in order to reduce the incidence of CIED-related infection. 